\title{
Reconfigurable control of flexible joint robot with actuator fault and uncertainty
}

\author{
Aymen Elghoul $^{*, * *}$, Adel Tellili*,**, Mohamed Naceur Abdelkrim ${ }^{*, * * *}$
}

\begin{abstract}
This paper presents the fault tolerant control (FTC) of a flexible joint robot using singular perturbation method in order to compensate for the lost performance due to the occurrence of actuator fault and the uncertainty. This FTC is based on Lyapunov redesign principle. The singular perturbation method is used to reduce the dynamic model of the flexible joint robot in a fast and slow subsystem. The time scale reduction of the flexible joint model is carried out when their joint stiffness is large enough and the singular perturbation parameter is set to zero. The fault-tolerant control structure in this paper is based on two parts. The first term described the composite control for the system without defect and without uncertainty which represents the sum between slow and fast controllers. While the second term of the fault tolerant command describes additive control designed to compensate for the fault effect of the actuator on the uncertain system. The additive approach is based on the Lyapunov theorem, which guarantees asymptotic stability despite the presence of actuator defects and the parametric uncertainty. The theoretical results are applied on a robot manipulator with a single flexible joint.
\end{abstract}

K e y w o r d s: uncertain flexible joint robot manipulators, singular perturbation method, additive fault tolerant control, actuator defect, Lyapunov theory

\section{Introduction}

In order to achieve good performance in the field of robot control, the first researchers have focused on studying the influence of motor dynamics. However, in 1985 Sweet and Good in [1] stressed that flexibility of the robot joint must be taken into account in robot modeling and control if high tracking performance is required. In 1987, Spong proposed in [2] a simplified model for the flexible articulation of the robot. After this, a large number of theoretical and experimental investigations were carried out on the control of robots with flexible joints: Singular perturbation and integral variety, feedback linearization, cascade system and integral backstepping control, PD control, adaptive control, robust control, neural network control, fuzzy control and some other commands [3-4].

The robot manipulators with flexible joints may be considered low elasticity systems if their joint stiffness is big enough. This characteristic allows us to transform the model of the flexible joint into a system with two time scales using the singular perturbation technique. The two time scales include a fast time scale (FTS) and a slow time scale (STS). The singular perturbation technique (SPT) is widely applied in the system which can be divided into a fast subsystem and a slow subsystem [5]. Thanks to the advantage of model reduction, this technique is capable of decomposing a higher order system into two systems of lower order [6-8]. The main idea of using the SPT technique in robots control is based on the addition of a simple correction term to the control law for robots with rigid bodies in order to attenuate the elastic oscillation at the joint of the robot. However, this method can be used only in robotic systems with weak joint flexibilities, where the dynamics of flexible joints is much faster than the dynamics of rigid bodies [9-10].

The singular perturbation model for the same system is not unique which involves several kinds of control schemes. Spong presented in [11] a control design that models elastic articulation forces as fast variables and body variables as slow variables. Ge proposed in [12] a new adaptive controller based on the SPT technique, which uses only feedback from position and velocity. In this control, engine tracking errors are modeled as fast variables and body variables are modeled as slow variables. As a result, the dynamics of the slow time scale and the resulting control laws are also different from those given by Spong.

Like other systems, the manipulator robot with flexible hinges can be infected by defects that can attack the components of the system (controller, actuator, sensor, etc), it is necessary to take into consideration the existence of these defects and to act on the control law so as to compensate for it and permitting the system to accomplish its mission. This type of control is called fault tolerant control (FTC). It covers all control strategies capable of preserving to the best of certain performances fixed by the operator such as stability, precision and speed; Not

* Research Unit MACS, Higher National Engineering School at Gabès, Tunisia, ** Higher Institute of Technological Studies at Djerba (ISETJB), Tunisia, aymengoul@hotmail.fr, adel.tellili@isetjb.rnu.tn, National Engineering School at Gabès, University of Gabès, Tunisia, naceur.abdelkrim@enig.rnu.tn 
only in normal operation but also in faulty mode, to improve and preserve the security and the ease of the processes and operators [13-17]. In the literature, there are two approaches to fault tolerant control. The first one is passive. In this approach, robust control techniques are used so that the closed system becomes insensitive to a known set of defects $[18,19]$. The defects are then considered in the initial design of the control system. The controller is therefore robust to predefined faults [19]. The second kind of fault tolerant control is the active approach which reacts to the various defects by reconfiguring the control law online so as to maintain the stability and the performances of the system [19,20]. In order to develop fault-tolerant controllers for flexible joint manipulators several approaches are presented in the literature. Kotosaka et al in [21] presented an FTC scheme for a robot manipulator that rearranges the trajectory in the event of an actuator fault, when the actuator no longer functions. Goel et al in [22] developed a method to minimize the peak error of the final effectors velocity in the event of a defect for manipulator robot. Lewis and Maciejewski in [23] developed an FTC scheme for a manipulator robot subjected to locked joint failures for the robot manipulator can reach critical points in the faulty case. Ting et al presented in [24] a sliding mode control and parameter adaptation laws which minimize errors caused by the fault. Izumikawa et al in [25] developed an FTC scheme based on the gain change so that stability and system performance are maintained in the event of a sensor fault for flexible robot.

The researchers are also interested in the uncertainty effect on the robotic system. The model uncertainties lead to a wrong system model and affect the accuracy of the system parameters like the mass and the inertia. That can influence the system performance and stability. Yang and Tan in [26] designed a sliding mode boundary controller for a single flexible-link manipulator based on adaptive radial basis function ( $\mathrm{RBF}$ ) neural network in presence of the uncertainties and external disturbances. In [27] Xu developed the adaptive sliding mode control based on the neural networks for flexible-joint robot with compound uncertainty. The model considered by authors is extracted using singular perturbation (SP) theory. Liu and Huang in [28] designed a robust adaptive controller based on singularly perturbed method, for flexible-joint manipulators with unknown upper bounds of parameter uncertainties and external disturbances. In [29] Asadi and Shandiz presented an adaptive tracking control for a class of flexiblejoint manipulators in the presence of parametric uncertainties. Furthermore, some authors like [30] considered the problem of input saturation for flexible manipulator and designed and adaptive control scheme to overcome the problem. In practice, the robotic system can present at the same time uncertainties such as (parametric uncertainties, dynamics modeling uncertainties, compound uncertainty ... ) and actuator faults like (arm collision, because this defect is one of the common faults on which a robot collides with an object or a human, and it may leads to serious damage or injury [33]). To the best our knowledge, there have been no studies to design FTC schemes to control a flexible manipulator with actuator fault and parametric uncertainty based on the singular perturbation approach.

The contribution of this paper is to consider the flexible joint robot system with real parameter as a two time scale system model to design a FTC in presence of actuator fault (collision defect) and uncertainty. The control scheme consists of two parts, a composite control designed to deal with the nominal case of a flexible joint robot (without failures and without uncertainty parameter) and an additive control that allows to compensate for the uncertainty of the system and the effect of the actuator failure; which makes the controller to guarantee the stability of system not only in case of collision failure, but also in case of parametric uncertainty.

\section{Singularly perturbed model of a flexible joint manipulator}

The method of the Lagrange which takes into account the potential energy of the flexible transmissions, allows leading to the dynamic model. In the following, we present, firstly, the dynamic model of the robot, then an order reduction using singular perturbation theory to simplify the controller design.

\subsection{Dynamic model of a flexible joint manipulator}

The simplified dynamics of a multi-axis flexible joint robot can be written as [25]

$$
\begin{gathered}
M(q) \ddot{q}+C(\ddot{q}, \dot{q}) \dot{q}+G(q)=K(\theta-q), \\
J \ddot{\theta}+K(\theta-q)=u
\end{gathered}
$$

where $q \in \mathbb{R}^{n}$ and $\theta \in \mathbb{R}^{n}$ represent the link angles and motor angles respectively, $M(q) \in \mathbb{R}^{n \times n}$ is a positively definite, symmetric inertia matrix of the robot links (including the motor masses), $C(q, \dot{q}) \dot{q} \in \mathbb{R}$ are the centripetal and Coriolis force, $G(q) \in \mathbb{R}$ is the gravity force vector and $K \in \mathbb{R}^{n \times n}$ is a diagonal matrix representing the joint stiffness. For notational simplicity we will assume that all joint stiffness constants are the same. $J \in \mathbb{R}^{n \times n}$ is the matrix of the moments of the inertia of the motors and $u \in \mathbb{R}^{n}$ is the exogenous input torque vector.

\subsection{Order reduction by $S P T$}

For the use of the singular perturbation theory in case of the flexible joint manipulator model, we follow the approach proposed by [35]. The reduced flexible model (1-2) can be put into a singularly perturbed form by introducing the small parameter $\varepsilon$ such that the stiffness is expressed as $K=K_{1} / \varepsilon^{2}$, where $K$ and $K_{1}$ are in order of $O\left(1 / \varepsilon^{2}\right)$ and $O(1)$ respectively. We define $z=K(\theta-q)$ 
where $z$ represents the torque vector transmitted through the elastic joints. Then (1) and (2) can be rewritten as

$$
\left\{\begin{array}{l}
M(q) \ddot{q}+C(q, \dot{q}) \dot{q}+G(q)=z, \\
\varepsilon^{2} J \ddot{z}+K_{1} z=K_{1}(u-J \ddot{q}) .
\end{array}\right.
$$

In order to write the complete system (3) in standard singular perturbation form [32], it is necessary to solve for $\ddot{q}$ in first equation of system (3) as

$$
\begin{aligned}
& \ddot{q}=\frac{\varphi(z, q, \dot{q})}{M(q)} \\
& \text { with } \varphi(z, q, \dot{q})=[z-C(q, \dot{q}) \dot{q}-G(q)]
\end{aligned}
$$

Using (4), we can further write the second equation of system (3) as

$$
\varepsilon^{2} J \ddot{z}+K_{1} z=K_{1}\left(u-J M(q)^{-1} \varphi(z, q, \dot{q})\right)
$$

The standard singular perturbation form results by combining (4) and (5) with the choice of state variables: $x=\left[\begin{array}{ll}q & \dot{q}\end{array}\right]^{\top}, y=\left[\begin{array}{ll}z & \varepsilon \dot{z}\end{array}\right]^{\top}$ so that

$$
\begin{cases}\dot{x}=f(x, y), & x \in \mathbb{R}^{2 n}, \\ \varepsilon \dot{y}=g(x, y, u), & y \in \mathbb{R}^{2 n}, \quad u \in \mathbb{R}^{n}\end{cases}
$$

where

$$
\left\{\begin{array}{l}
f(x, y, u)=\left(\begin{array}{c}
\dot{q} \\
M(q)^{-1} \varphi(z, q, \dot{q})
\end{array}\right) \\
g(x, y, u)=\left(\begin{array}{c}
\dot{z} \\
K_{1}\left(J^{-1} u-\ddot{q}-J^{-1} z\right)
\end{array}\right) .
\end{array}\right.
$$

Note that the system (6) is singularly perturbed, where $\left[\begin{array}{ll}q & \dot{q}\end{array}\right]$ and $\left[\begin{array}{ll}z & \dot{z}\end{array}\right]$ represent respectively the fast variables and the slow variables. Based on the results of the singular perturbation theory [32], the reduction procedure is carried out neglecting the duration of variation of the fast modes in front that of the dominant slow modes which determine the dynamics of the system. The slow model is obtained by considering that the fast variables in $z$ have reached their steady state, which is to assume that $\varepsilon=0$ in (6), leading to

$$
\bar{z}=\bar{u}-J \ddot{\bar{q}}
$$

where

$$
\bar{z}=\left.z\right|_{\varepsilon=0}, \quad \bar{q}=\left.q\right|_{\varepsilon=0}, \quad \bar{u}=\left.u\right|_{\varepsilon=0} .
$$

Substituting (8) into the first equation of (6) gives the following slow subsystem as

$$
\ddot{\bar{q}}(M(\bar{q})+J)+C(\bar{q}, \dot{\bar{q}}) \dot{\bar{q}}+G(\bar{q})=\bar{u}=u_{s}
$$

which is identical to the rigid robot model [36], where the subscript s stands for slow variables. On the other hand, setting (10) in (5) yields the expression

$$
K_{1} \bar{z}=K_{1}\left(\bar{u}-J M(\bar{q})^{-1} \varphi(z, \bar{q}, \dot{\bar{q}})\right)
$$

The fast system will be analyzed with the boundary layer theory of singular perturbation method. In order to describe the fast system, a new variable $\tau=t / \varepsilon$ is introduced. Then, the boundary-layer system is written in terms of the variable $z_{f}=z-\bar{z}$, where the subscript $f$ stands for fast variables. Here $z$ is the torque of the spring, $\bar{z}$ defined by (8), is constant in the fast time scale $\tau$ and $z_{f}$ is the fast part.

Changing to the fast time scale $\tau$, and $z=\bar{z}+z_{f}$ which will be substituted into (11) with $\ddot{\bar{z}}=0$ and $\varepsilon=0$, gives the expression

$$
\begin{aligned}
& J \frac{\mathrm{d}^{2} z_{f}}{\mathrm{~d} \tau^{2}}+K_{1}\left(\bar{z}+z_{f}\right)= \\
& \quad K_{1}\left(u-J M(q)^{-1}\left[\left(\bar{z}+z_{f}\right)-C(q, \dot{q}) \dot{q}-G(q)\right]\right) .
\end{aligned}
$$

Using (8) and (10) (for $\bar{z}$ and $u_{s}$ ) (12) reduces to

$$
\frac{\mathrm{d}^{2} z_{f}}{\mathrm{~d} \tau^{2}}+K_{1}\left(J^{-1}+M(\bar{q})^{-1}\right) z_{f}=J^{-1} K_{1} u_{f}
$$

where $u_{f}=u-u_{s}$ is the control for the fast subsystem which is responsible for the dynamic response and $u_{s}$ for the steady-state response.

In view of the singular perturbation theory, a composite control structure can be considered. This control scheme consists of two terms $u_{s}$ and $u_{f}$ separately designed [11]

$$
u=u_{s}+u_{f} .
$$

The synthesis of the control $u_{s}$ of the slow subsystem is based on the quasi-static approximation of the system (10) and only affects the latter, while the control of the subsystem $u_{f}$ is aimed at stabilizing the fast subsystem (13). We typically search for $u_{s}=\left.u\right|_{s=0}=\bar{u}$ and $\left.u_{f}\right|_{s \rightarrow 0}=0$. Thus, $u_{s}$ can be chosen from the methods for controlling rigid robots.

\section{FTC scheme for flexible-joint robotic manipulators}

Design a slow control $u_{s}(t)=R_{s}(x)$ for the reduced system (10) such that $x=0$ is its unique asymptotically stable equilibrium in $B_{x} \subset R^{n 1}$, and where a Lyapunov function $L_{s}(x): R_{n} \rightarrow \mathbb{R}^{+}$exists, guaranteeing, for all $x \in D_{x},[5]$

$$
\begin{aligned}
& \frac{\partial L_{s}}{\partial x}\left[\rho_{1}(x, \bar{z})+\sigma_{1}(x, \bar{z}) u_{s}\right] \leq-p \Psi^{2}(x), \quad p>0 \\
& \text { where, } \sigma_{1}(x, \bar{z})=\left(M\left(q_{s}\right)+J\right)^{-1} \\
& \left.\left.\rho_{1}(x, \bar{z})=\sigma_{1}(x, \bar{z})\left(-C\left(q_{s}, \dot{q}_{s}\right) \dot{q}_{s}\right)-G\left(q_{s}\right)\right)\right)
\end{aligned}
$$

and $\Psi(x)$ is a positive definite function with $\Psi(0)=0$.

Knowing that $u_{s}(t)=R_{s}(x)$, a fast control $u_{f}(t)=$ $R_{f}(x, y)$ which satisfies $R_{f}\left(x, T\left(x, R_{s}(x)\right)\right)=0$ is designed to stabilize asymptotically the fast dynamics, such that the equilibrium $\bar{y}=\bar{z}=T\left(x, u_{s}\right)$ of the closed-loop boundary layer system (13) is supposed asymptotically stable. For this subsystem, we consider a positive-definite 


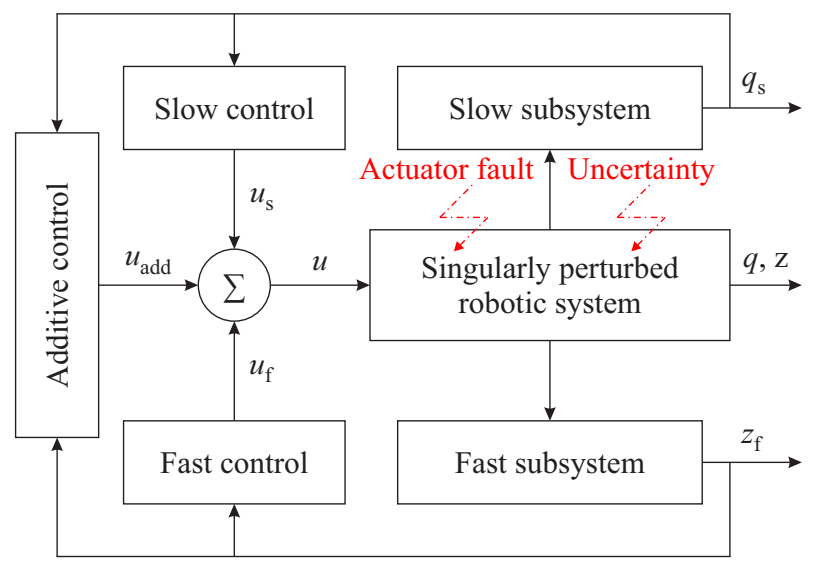

Fig. 1. Functional diagram of the fault-tolerant control of a singularly perturbed uncertain robotic system

Lyapunov function $L_{f}(x, y): R^{n 1} \times R^{n 2} \rightarrow \mathbb{R}^{+}$such that for all $(x, y) \in D_{x} \times D_{y}$

$$
\frac{\partial L_{f}}{\partial z_{f}}\left[\rho_{2}(x, y)+\sigma_{2}(x, y)\left(R_{s}+R_{f}\right)\right] \leq-c \phi^{2}\left(z_{f}\right), c>0
$$

where $\rho_{2}(x, y)=\left(K_{1} M(q)^{-1}[\varphi(z, q, \dot{q})]-K_{1} J^{-1} z\right)$, $\sigma_{2}(x, y)=J^{-1} K_{1}, \phi(x, y)$ is a continuous positivedefinite scalar function that satisfies $\phi(0)=0$ and $\varphi(z, q, \dot{q})$ is given in (4).

Since the control input to system (6) is the composite control, it is necessary to ensure the asymptotic stability property of the system. To show how this can be achieved, we construct a weighted sum of the Lyapunov functions. Define this function as [5]

$$
H\left(x, z_{f}\right)=(1-\alpha) L_{s}(x)+\alpha L_{f}\left(x, z_{f}\right), 0<\alpha<1,
$$

where, $L_{s}$ and $L_{f}$ are the Lyapunov functions of the slow subsystem (10) and fast subsystem (13), respectively, and $\alpha$ is a free parameter. The derivative of this composite Lyapunov function along (6) gives

$$
\begin{gathered}
\dot{H}=\frac{\alpha}{\varepsilon} \frac{\partial L_{f}\left(x, z_{f}\right)}{\partial z_{f}}\left[\rho_{2}(x, z)+\sigma_{2}\left(x, z_{f}\right) u\right] \\
\quad+(1-\alpha) \frac{\partial L_{s}(x)}{\partial x}\left[\rho_{1}\left(x, h\left(x_{1}, u_{s}\right)\right)\right. \\
\left.+\sigma_{1}\left(x, h\left(x, R_{s}(x)\right)\right) u_{s}\right]+M\left(x, z_{f}, u, u_{s}\right) .
\end{gathered}
$$

The terms in (18) represent respectively $\dot{L}_{f}$ and $\dot{L}_{s}$ along the trajectories of the fast and slow subsystems respectively. Based on the two equations (15) and (16), we can conclude that the terms $\dot{L}_{f}$ and $\dot{L}_{s}$ in equation (18) are negative-definite. While the effect of the interconnection between the fast and slow dynamics is presented in the third term $M\left(x, z_{f}, u, u_{s}\right)$ which takes the following form

$$
\begin{array}{r}
M\left(x, z_{f}, u, u_{s}\right)=(1-\alpha) \frac{\partial L_{s}(x)}{\partial x}\left[\rho_{1}\left(x, z_{f}\right)+\sigma_{1}\left(x, z_{f}\right) u\right. \\
\left.-\rho_{1}\left(x, T\left(x, R_{s}(x)\right)\right)-\sigma_{1}\left(x, T\left(x, R_{s}(x)\right)\right) u_{s}\right] \\
+\alpha \frac{\partial L_{f}\left(x, z_{f}\right)}{\partial x}\left[\rho_{1}\left(x, z_{f}\right)+\sigma\left(x, z_{f}\right) u\right] .
\end{array}
$$

For this term to be negligible it is necessary that the parameter of singular perturbation parameter $\varepsilon$ remains weak [31]. According to (15) and (16), we can reorganize equation (18) in the following form

$$
\frac{\partial H\left(x, z_{f}\right)}{\partial t} \leq-\nu\left(x, z_{f}\right)
$$

where $\nu\left(x, z_{f}\right)=p \Psi^{2}(x)+\frac{\alpha}{\varepsilon} c \phi^{2}\left(z_{f}\right)$ is positive definite and $H\left(x, z_{f}\right)$ is a Lyapunov function. Therefore, within a predetermined range of parameter $\varepsilon$, it can be concluded that the system (6) is asymptotically stable at the origin due to the application of composite control (14) [32-35].

After stabilization of the slow and fast subsystems in $D_{x} \times D_{y}$ and we can access states $(x, y)$, we are looking for a fault-tolerant control used to keep the singularly perturbed system stable at the origin even in the presence of actuator faults and parametric uncertainty.

The singularly perturbed system for flexible Joint robots (6), when the actuator affected by an additive defect and in the presence of uncertainty, the system can be rewritten in the following form

$$
\left\{\begin{array}{l}
\ddot{q}=\frac{\varphi(z, q, \dot{q})}{M(q)}+\Delta_{1}(q, \dot{q}), \\
\varepsilon \ddot{z}=K_{1} J^{-1}((u+F(t, x, y)) \\
\left.-J M(q)^{-1} \varphi(z, q, \dot{q})-z\right)+\Delta_{2}(q, \dot{q}) .
\end{array}\right.
$$

Assumption 1. There exists a known positive constant $D(x, y)>\|F(x, y)\|, D(x, y)>\left\|\Delta_{1}(q, \dot{q})\right\|$ and $D(x, y)>\left\|\Delta_{2}(q, \dot{q})\right\|$ for all $t \geq 0$. Thus, the fault tolerant control designed to stabilize the faulty system (20) is presented as [5]

$$
u=u_{\text {nom }}+u_{\text {add }},
$$

$u_{\text {nom }}$ and $u_{\text {add }}$ are respectively the nominal composite control designed to stabilize the faulty system and the additive control designed to reduce the effect of fault and uncertainty on the system. A block scheme representation of the implementation of the fault tolerant control is given in Fig. 1.

To ensure the asymptotic stability of the uncertain system (20) with actuator fault by the control (21), we consider a positive definite Lyapunov function

$$
H\left(\varepsilon, x, z_{f}\right)=(1-\alpha) L_{s}+\varepsilon \alpha L_{f} .
$$

The derivative of this function is

$$
\begin{gathered}
\frac{\partial H\left(\varepsilon, x, z_{f}\right)}{\partial t}=\alpha \frac{\partial L_{f}}{\partial z_{f}}\left[\rho_{2}+\sigma_{2}\left(u_{\mathrm{nom}}+u_{\mathrm{add}}+F\right)+\Delta_{2}\right] \\
+(1-\alpha) \frac{\partial L_{s}}{\partial x}\left[\rho_{1}+\sigma_{1}\left(u_{\mathrm{nom}}+u_{\mathrm{add}}+F\right)+\Delta_{1}\right] \\
+\alpha \varepsilon \frac{\partial L_{f}}{\partial x}\left[\rho_{1}+\sigma_{1}\left(u_{\mathrm{nom}}+u_{\mathrm{add}}+F\right)+\Delta_{1}\right]
\end{gathered}
$$


The separation of the terms which depend on the nominal and the additive controls makes (23) to be

$$
\begin{array}{r}
\frac{\partial H}{\partial t}=\alpha \frac{\partial L_{f}}{\partial z_{f}}\left(\rho_{2}+\sigma_{2} u_{\text {nom }}\right)+(1-\alpha) \frac{\partial L_{s}}{\partial x}\left(\rho_{1}+\sigma_{1} u_{\text {nom }}\right) \\
+\alpha \varepsilon \frac{\partial L_{f}}{\partial x}\left(\rho_{1}+\sigma_{1} u_{\text {nom }}\right)+(1-\alpha) \frac{\partial L_{s}}{\partial x} \sigma_{1}\left(u_{\text {add }}+F\right) \\
+(1-\alpha) \frac{\partial L_{s}}{\partial x} \Delta_{1}+\alpha \frac{\partial L_{f}}{\partial z_{f}} \sigma_{2}\left(u_{\text {add }}+F\right)+\alpha \frac{\partial L_{f}}{\partial z_{f}} \Delta_{2} \\
+\alpha \varepsilon \frac{\partial L_{f}}{\partial x} \sigma_{1}\left(u_{\text {add }}+F\right)+\alpha \varepsilon \frac{\partial L_{f}}{\partial x} \Delta_{1} .
\end{array}
$$

We take $\frac{\partial L_{s}}{\partial x}=S_{1}^{\top}, \frac{\partial L_{f}}{\partial z_{f}}=S_{2}^{\top}, \frac{\partial L_{f}}{\partial x}=S_{3}^{\top}$ and, considering (19), (24) becomes

$$
\begin{aligned}
\frac{\partial H}{\partial t} \leq-\nu+ & (1-\alpha) S_{1}^{\top} \sigma_{1}\left(u_{\text {add }}+F\right) \\
+ & \alpha S_{2}^{T} \sigma_{2}\left(u_{\text {add }}+F\right)+\alpha \varepsilon S_{3}^{\top}\left(u_{\text {add }}+F\right) \\
& +(1-\alpha) S_{2}^{\top} \Delta_{2}+\alpha S_{2}^{\top} \Delta_{2}+\alpha \varepsilon S_{3}^{\top} \Delta_{1} .
\end{aligned}
$$

We can rewrite the inequality (25) in the following form

$$
\begin{aligned}
\dot{H} \leq-\nu+ & {\left[(1-\alpha) S_{1}^{\top} \sigma_{1}+\alpha S_{2}^{\top} \sigma_{2}+\alpha \varepsilon S_{3}^{\top} \sigma_{1}\right] F } \\
+[ & \left.(1-\alpha) S_{1}^{\top} \sigma_{1}+\alpha S_{2}^{\top} \sigma_{2}+\alpha \varepsilon S_{3}^{\top} \sigma_{1}\right] u_{\mathrm{add}} \\
& +(1-\alpha) S_{1}^{\top} \Delta_{1}+\alpha S_{2}^{\top} \Delta_{2}+\alpha \varepsilon S_{3}^{\top} \Delta_{1} .
\end{aligned}
$$

The use of singular perturbation technique by putting the singular perturbation parameter $\varepsilon$ to zero and using Assumption 1, the following expression is obtained

$$
\begin{aligned}
\left.\dot{H}\right|_{\varepsilon=0} \leq-\nu+ & {\left[\alpha S_{2}^{\top} \sigma_{2}+(1-\alpha) S_{1}^{\top} \sigma_{1}\right] u_{\text {add }} } \\
& +D\left\|\alpha S_{2}^{\top} \sigma_{2}+(1-\alpha) S_{1}^{\top} \sigma_{1}\right\| \\
& +D\left(\left\|(1-\alpha) S_{1}^{\top}\right\|+\left\|\alpha S_{2}^{\top}\right\|\right)
\end{aligned}
$$

where $\nu\left(t, x, z_{f}\right)$ used in (19) is positive definite, the second term $u_{\text {add }}$ in the equation above designates the effect of the additive control. Whereas the last terms designate, respectively, the fault $F(t, x, y)$ and the uncertainties $\Delta_{1}$ and $\Delta_{2}$. To ensure asymptotic stability the derivative of the Lyapunov function (27) must be negative, the additive control $u_{\text {add }}$ takes then the following form

$$
\begin{aligned}
& u_{\text {add }}=-D \frac{(1-\alpha) S_{1}^{\top} \sigma_{1}+\alpha S_{2}^{\top} \sigma_{2}}{\left\|(1-\alpha) S_{1}^{\top} \sigma_{1}+\alpha S_{2}^{\top} \sigma_{2}\right\|^{2}} \times \\
& {\left[\left\|(1-\alpha) S_{1}^{\top} \sigma_{1}+\alpha S_{2}^{\top} \sigma_{2}\right\|+\left\|(1-\alpha) S_{1}^{\top}\right\|+\left\|\alpha S_{2}^{\top}\right\|\right] .}
\end{aligned}
$$

So, under the effect of the fault tolerant control (28), we can conclude that the uncertain system in the presence of fault $(20)$ is asymptotically stable at the origin $(x, y)=$ $(0,0)$.

Using the equation (28), the system (20) is rewritten in the following form

$$
\left\{\begin{array}{l}
\ddot{q}=\frac{\varphi(z, q, \dot{q})}{M(q)}+\Delta_{1}(q, \dot{q}), \\
\varepsilon \ddot{z}=K_{1} J^{-1}\left(\left(u+u_{\text {add }}+F(t, x, y)\right)\right. \\
\quad-J M(q)^{-1}(\varphi(z, q, \dot{q})-z)+\Delta_{2}(q, \dot{q}) .
\end{array}\right.
$$

The discontinuity of this control causes the chattering phenomenon that can excite the instability of the system. To overcome this problem, we apply the idea of the boundary layer method, using a continuous approach.

\section{A design example}

In this section, a simulations example is presented to show the efficiency and the performance of the studied reconfigurable control scheme. Toward this end, consider the single link flexible joint manipulator, represented schematically by Fig. 2. This robot can be expressed by the following nonlinear differential equations [35]

$$
\left\{\begin{array}{l}
I \ddot{q}+M g l \sin (q)=K(\theta-q), \\
J \ddot{\theta}+K(\theta-q)=u
\end{array}\right.
$$

where, $q$ and $\theta$ are the link and motor angles respectively, $I$ is the link inertia, $J$ being the inertia of motor, $K$ is the spring stiffness, $u$ is the input torque, and $M$ and $L$ are the mass and length of link respectively.

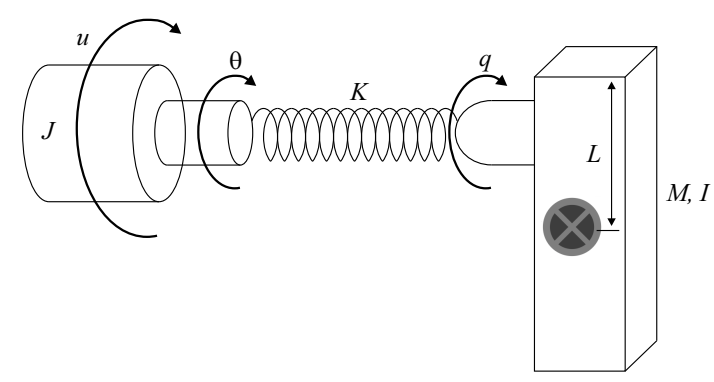

Fig. 2. Single-link flexible joint robot

The robot model with the flexible joint (30) can be put in a singularly perturbed form by introducing the parameter $\varepsilon$ such that $(3)[2,25]$

$$
\left\{\begin{array}{l}
\ddot{q}=\frac{-M g L \sin (q)+z}{I} \\
\varepsilon \dot{z}=\frac{M g L \sin (q)-z}{q} I+\frac{u-z}{J} .
\end{array}\right.
$$

The slow model is obtained by assuming $\varepsilon=0$ in (31). It takes the following form

$$
\ddot{q}=(I+J)^{-1}\left(u_{s}-M g L \sin (q)\right) .
$$

We seek to find the slow control $u_{s}$ so that the reduced slow system (32) becomes asymptotically stabilizing about the origin. By inspection, we find that 

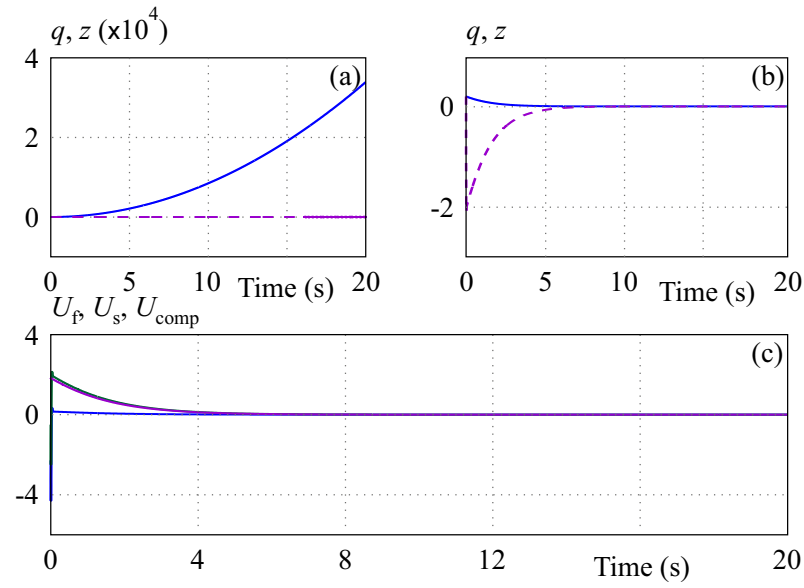

Fig. 3. Time evolution of states and controls in absence of fault and uncertainty: (a) - q (-) and z (-) in open-loop, (b) - evolution of states in closed-loop with composite control, (c) - fast (-.), slow $(-)$ and composite (-) controllers

$u_{s}=M g L \sin (q)-\Psi(x)$ stabilizes exponentially the origin, where $\Psi(\cdot)$ is a scalar function. In order to analyze the stability of the slow subsystem, the following slow Lyapunov function is introduced

$$
L_{s}(q, \dot{q})=\frac{1}{2}\left[\dot{q}^{\top} \dot{q}+q^{\top} q\right]
$$

The reduced fast subsystem for (31), in the fast time scale, is defined as

$$
\frac{\mathrm{d}^{2} z_{f}}{\mathrm{~d} \tau^{2}}=\frac{M g L}{I} \sin (q)-\left(\frac{1}{I}+\frac{1}{J}\right) z_{f}+\frac{1}{J}\left(u_{s}+u_{f}\right) .
$$

The control of the fast subsystem $u_{f}$ is expressed as

$$
u_{f}=z_{f}-K_{v} \dot{z}_{f}
$$

where, $K_{v}$ is a constant. This approach can be easily deducted, and guarantees the asymptotic stability of the slow subsystem (34) in a closed loop. The following Lyapunov function is proposed

$$
L_{f}\left(z_{f}, \dot{z}_{F}\right)=\frac{1}{2} \dot{z}_{f} \dot{z}_{f}^{\top}+\frac{1}{2} z_{f}\left(I^{-1}+J^{-1}\right) z_{f}^{\top} .
$$

In order to guarantee the stability of the singularly perturbed system without defect, a composite control structure with two terms can be considered. A slow control $u_{s}$ to stabilize the slow subsystem (rigid robot model) and a fast control $u_{f}$ designed to damp the elastic oscillations at the joints. The composite control becomes

$$
\begin{aligned}
u_{\mathrm{comp}}=u_{s}(q, \dot{q}, t)+u_{f}\left(z_{f}, \dot{z}_{f}\right) & \\
& =M g L \sin (q)-\Psi(x)+z_{f}-K_{v} \dot{z}_{f} .
\end{aligned}
$$

The resulting closed-loop overall system, after substituting of (37) in (31) is

$$
\left\{\begin{array}{l}
\ddot{q}=\frac{-M g L}{I} \sin (q)+\frac{1}{I} z, \\
\varepsilon \ddot{z}=\frac{M g L}{I} \sin (q)-\left(\frac{1}{I}+\frac{1}{J}\right) z+\frac{1}{J} u_{\mathrm{comp}}
\end{array}\right.
$$

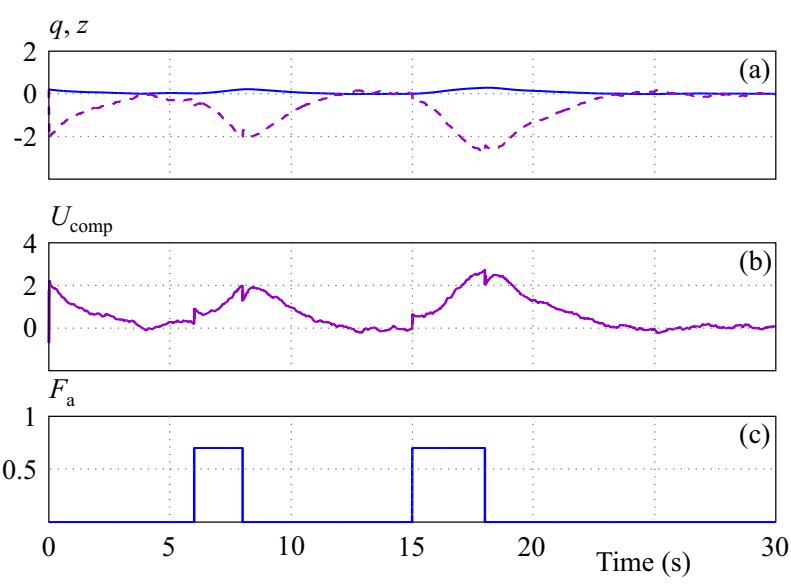

Fig. 4. Evolution of states and composite control with actuator fault and parametric uncertainty: (a) - q (-) and $\mathrm{z}(-)$ regulation by the composite control, (b)- composite control. (c) - fault of collision

where $q \in \mathbb{R}^{n}$ and $z \in \mathbb{R}^{n}$ represent the link angles and elastic torque.

For the simulation, the parameters of the QUANSERs flexible joint manipulator system are selected as follows [37]: $g=9.81 \mathrm{~m} / \mathrm{s}^{2}, M=0.065 \mathrm{~kg}, L=0.419 \mathrm{~m}$, $I=3.8 \times 10^{-3} \mathrm{~kg} \cdot \mathrm{m}^{2}, \quad J=2.08 \times 10^{-3} \mathrm{~kg} \cdot \mathrm{m}^{2}, K=$ $1.3 \mathrm{~N} . \mathrm{m} / \mathrm{rad}$.

The first simulation presented in Fig. 3 shows the performance of the controls we have developed previously in the fault-free case. It is clear that the composite $u_{\text {comp }}$ asymptotically stabilizes the origin of the flexible-joint robotic system in (38). The initial conditions chosen for the state variables are $\left[\begin{array}{llll}q_{0} & \dot{q}_{0} & z_{0} & \dot{z}_{0}\end{array}\right]=\left[\begin{array}{llll}0.1 & 0.1 & 0.1 & 0.1\end{array}\right]$.

In order to verify the effectiveness of the control (37), two collision faults on the actuator $F_{a}$, see Fig. 4(c) are injected. The first one is generated between the time instants $6 \mathrm{~s}$ and $8 \mathrm{~s}$ (before reaching the steady state) and the second one between the time instants $15 \mathrm{~s}$ and $18 \mathrm{~s}$ (after reaching the steady state): A collision defect is one of the common faults on which a robot collides with an object or a human, and it may leads to serious damage or injury. The generated parametric uncertainty is $10 \%$ of the nominal value of the system parameters (38). Figure (4) shows that the composite control (37) becomes incapable of ensuring the convergence of the flexible-joint robotic system (38) in the presence of uncertainty and defect (see Fig. 4(a)). Hence the need for fault-tolerant control that eliminates the effect of fault and uncertainty from the states.

Then, in order to reduce the effect of the actuator fault and the uncertainty on the system, a fault tolerant approach will be introduced, according to equations (21) and (28), it takes the form

$$
\begin{aligned}
u=u_{\text {comp }}+u_{\text {add }}= & M g L \sin (q)-\Psi(x)+ \\
& z_{f}-K_{v} \dot{z}_{f}-D \frac{\zeta(x, y)\|\delta(x, y)\|}{\|\zeta(x, y)\|^{2}}
\end{aligned}
$$




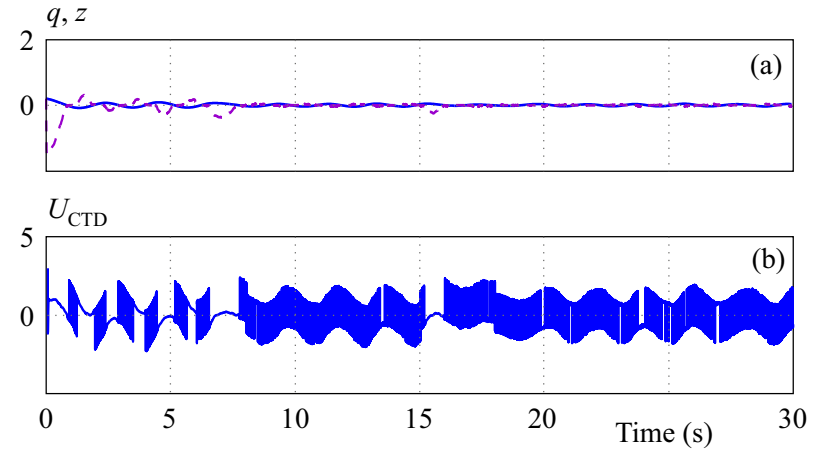

Fig. 5. Evolution of states and composite control with actuator fault and parametric uncertainty: (a) - q (-) and z (-) by the faulttolerant control, (b) - fault tolerant controller

where, $\zeta(\cdot)$ and $\delta(\cdot)$ are continuous scalar function. The application of this control gives Fig. 5 .

It can be seen from Fig. 5 that the control applied to the uncertain robotic system with faulty makes it capable of maintaining its performance even in the presence of uncertainty and collision defect. However, the corresponding controls are characterized by very fast switching causes the chattering phenomenon that can excite the instability of the system.

In order to reduce the chattering phenomenon, while maintaining the fault tolerant control performance, the saturation function will be used instead of the discontinuous function. The simulation results in Fig. 6(a) and (b) represent, respectively, the states and the controller after the attenuation of the chattering in the control law. We can see the disappearance of the chattering phenomenon and that the control which was applied to the faulty uncertain system makes it capable of maintaining its performance and states stay at the same equilibrium point.

\section{Conclusion}

In this paper we applied the singular perturbation control strategy for the case of uncertain flexible joint robot to ensure the stability of the global system, not only when the actuator is fault free but also in the presence of collision defects. Firstly, the flexible joint robot system is modeled using the singular perturbation method, it is divided into a slow and a fast subsystems. The fault tolerant control scheme has two main components. The composite part, assumed as a classic nominal control of the global system without considering the dynamic uncertainty and the fault. it is represented by the sum of the slow and fast sub-controllers. The second component is the additive part introduced in order to remove the fault effect in the actuator and the parametric uncertainty. This additive approach is based on the Lyapunov theorem, which guarantees asymptotic stability despite the presence of actuator defect and uncertainty.

A robot manipulator with a single flexible joint has been presented as an illustrative example, on which we applied the fault tolerant control method, it is shown that

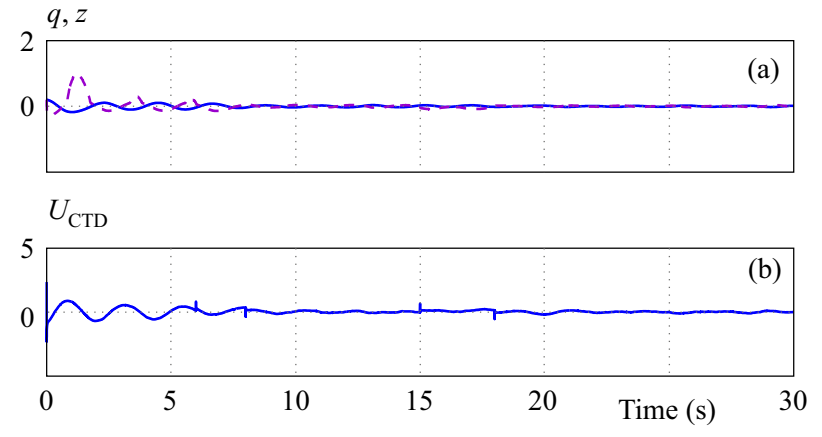

Fig. 6. Evolution of states: (a) - and fault tolerant control, (b) after the reduce of chattering phenomenon

the reconfigurable control was able to eliminates the effect of actuator fault and the uncertainty.

\section{REFERENCES}

[1] L. M. Sweet and M. C. Good, "Redefinition of the Robot Motion-Control Problem", IEEE Control Systems Magazine vol. 5, no. 3, pp. 18-24, 1985.

[2] M. Spong, "Modeling Control of Elastic Joint Robots", Journal of Dynamic Systems Measurement Control vol. 109, no. 4, pp. 310-319, 1987.

[3] M. Sayahkarajy, M. Zamri, and A. A. Mohd Faudzi, "Review of Modelling Control of Flexible-Link Manipulators", Journal of Systems Control Engineering vol 230, no, 8, pp. 861-873, 2016.

[4] I. Giorgio, A. Della Corte, and D. Del Vescovo, "Modelling Flexible Multi-Link Robots for Vibration Control: Numerical Simulations Real-Time Experiments", Journal of Mathematics Mechanics of Solids, 2017,.

[5] A. Tellili, A. Elghoul, and M. N. Abdelkrim, "Additive Fault Tolerant Control of Nonlinear Singularly Perturbed Systems Against Actuator Fault", Journal Electrical Engineering vol. 68, no. 1, pp. 68-73, 2017.

[6] Y. C. Liu, M. H. Jin, and H. Liu, "Singular Perturbation Control for Flexible Joint Manipulator based on Flexibility Compensation", Jiqiren Robot vol. 30, no. 5, pp. 460-466, 2008.

[7] A. Massoum, A. Meroufel, P. Wira, and M. A. Fellah, "Fuzzy Control of the Permanent Magnet Synchronous Machine Singularly Perturbed Fed by a Three Level Inverter", Journal Electrical Engineering, vol. 63 no. 3, pp. 186-190, 2012.

[8] C. T. Kiang, A. Spowage, and C. K. Yoong, "Review of Control Sensor System of Flexible Manipulator", Journal of Intelligent \& Robotic Systems vol. 77, no. 1, pp. 187-213, 2015.

[9] K. Wang, "Modélisation d'un Robot Manipulateur en vue de la Commande Robuste en Force utilisé en Soudage FSW", Doctoral thesis, Ecole nationale supérieure d'arts et métiers, 2016.

10] J. C. Cambera and V. Feliu-Batlle, "Input-State Feedback Linearization Control of a Single-Link Flexible Robot Arm Moving under Gravity and Joint Friction", Journal of Robotics Autonomous Systems, vol. 88, pp. 24-36, 2017.

11] M. K. Spong, and P. Khorasani, "An Integral Manifold Approach to the Feedback Control of Flexible Joint Robots", IEEE Journal of Robotics Automation vol. 3, no. 4, pp. 291-300, 1987.

[12] S. Ge, "Adaptive Controller Design for Flexible Joint Manipulators", Automatica vol. 32, no. 2, pp. 273-277, 1996.

[13] D. Bustan, S. K. H. Sani, and N. Pariz, "Adaptive Fault-tolerant Spacecraft Attitude Control Design with Transient Response Control", IEEE/ASME Transactions on Mechatronics, vol. 19, no. 4, pp. 1404-1411, 2014. 
[14] Y. Wang, P. Zhou, Q. Wang, and D. Duan, "Reliable Robust Sampled-Data H-infinity Output Tracking Control with Application to Flight Control", Information Technology Control vol. 43, no. 2, pp. 175-182, 2014.

[15] C. T. Kiang, A. Spowage, and C. K. Yoong, "Review of Control Sensor System of Flexible Manipulator", Journal of Intelligent \& Robotic Systems vol. 77, no. 1, pp. 187-213, 2015.

[16] M. Boukhnifer, "Active Fault Tolerant Control for Ultrasonic Piezoelectric Motor", Journal of Electrical Engineering vol. 63, no. 3, pp. 224-232, 2012.

[17] M. S. Khireddine and A. Boutarfa, "Reconfigurable Control for a Scara Robot using RBF Networks", Journal of Electrical Engineering vol. 61 no. 2, pp. 100-106, 2010.

[18] F, Guenab, "Contribution aux systèmes tolérants aux défauts: synthèse dune méthode de reconfiguration et/ou de restructuration intégrant la fiabilité des composants", Doctoral thesis, Université de Henri Poincaré, 2007.

[19] B. Jiang, M. Staroswiecki, and V. Cocquempot, "Fault Accommodation for Nonlinear Dynamic Systems", IEEE Transactions on Automatic Control vol. 51, no. 9, pp. 1578-1583, 2006.

[20] M. Boukhnifer, "Active Fault Tolerant Control for Ultrasonic Piezoelectric Motor", Journal of Electrical Engineering vol. 63, no. 3, pp. 224-232, 2012.

[21] S. Kotosaka, H. Asama, H. Kaetsu, I. Endo, K. Sato, and R. Nakayama, "Fault Tolerance of a Functionally Adaptive Robust Manipulator", IEEE Int. Conf, on Intelligent Robots Systems, pp. 294-300, 1993.

[22] M. Goel et al, "Failure Tolerant Teleportation of a Kinematically Redundant Manipulator: an Experimental Study", IEEE Transactions on Systems vol. 30, no. 6, pp. 758-765, 2003.

[23] C. L. Lewis and A. A. Maciejewski, "Fault Tolerant Operation of Kinematically Dedundant Manipulators for Locked Joint Failures", IEEE Trans. Robotics Automat vol. 13, no. 4, pp. 622-629, 1997.

[24] Y. Ting, S. Tosunoglu, and B. Fernandez, "Control Algorithms for Fault Tolerant Robots", IEEE Int. Conf, on Robotics Automation San Diego, CA, USA, 1994.

[25] Y. Izumikawa, K. Yubai, and T. Hori, "Vibration Suppression Control of Flexible Arm Robot by PD Gain Switching Considering Sensor Failure", IEEE Int. Conf, on Industrial Technology Bangkok, pp. 684-689, 2002.

[26] H. J. Yang and M. Tan, "Sliding Mode Control for Flexible-Link Manipulators Based on Adaptive Neural Networks", International Journal of Automation Computing vol. 15, no. 2, pp. 239-248, 2018.

[27] B. Xu, "Composite Learning Control of Flexible-Link Manipulator Using NN and DOB", IEEE Transactions on Systems Man and Cybernetics: Systems vol. 48, no. 11, pp. 1979-1985, 2017.

[28] H. Liu and Y. Huang, "Robust Adaptive Output Feedback Tracking Control for Flexible-Joint Robot Manipulators based on Singularly Perturbed Decoupling", Robotica vol. 36, no. 6, pp. 822-838, 2018.

[29] M. Asadi and H. T. Shandiz, "Time Scale Separation in Control of a Single-Link Flexible-Joint Robot Manipulator", IMA Journal of Mathematical Control Information vol. 35, no. 2, pp. 357-373, 2016.

[30] Z. Liu, J. Liu, and W. Hu, " Adaptive Boundary Control of a Flexible Manipulator with Input Saturation", International Journal of Control vol. 89, no. 6, pp. 1191-1202, 2015.

[31] H. K, Khalil, "Nonlinear Systems", Prentice Hall, United States of America, 2002.
32] P. Y. kokotovic, H. K. khalil and J. Oreilly, "Singular Perturbation Methods in Control-Analysis and Design", Harcourt Brace Jovanovich publishers, Academic press, United States of America, 1999

33] A. Saberi and H. K. Khalil, "Stabilization Regulation of Nonlinear Singularly Perturbed Systems-Composite Control", IEEE Transactions on Automatic Control vol. 30, no. 8, pp. 739-747, 1985.

[34] A. Narang-Siddarth and J. Valasek, "Nonlinear Time Scale Systems in Standard and Nonstandard Forms - Analysis and Control", Siam, United States of America, 2014.

35] M. W. Spong, "Adaptive Control of Flexible Joint Manipulators: Comments on Two Papers", Automatica vol. 31, no. 4, pp. 585-590, 1995.

36] M. W. Spong, "Adaptive Control of Flexible Joint Manipulators", Journal of Systems \& Control Letters vol. 13, no. 1, pp. 15-21, 1989.

[37] Y. Ouyang, W. He, and X. Li, "Reinforcement Learning Control of a Single Link Flexible Robotic Manipulator", IET Control Theory \& Applications vol. 11, no. 9, pp. 1426-1433, 2017.

[38] Y. N. Cho, J. T. Hong, and H. J. Kim, "Neural Network Based Adaptive Actuator Fault Detection Algorithm for Robot Manipulators", Journal of Intelligent \& Robotic Systems, pp. 1-11, 2018.

Received 23 February 2019

Aymen Elghoul was born in Tunisia in 1987, he graduated from the National School of Engineers in Carthage, Tunisia, in 2013. He received the Master degree from the Higher Institute of Industrial Systems of Gabes in 2015. He is currently an Assistant at the Higher Institute of Technological Studies at Djerba. He is member at research Laboratory on Modeling, Analysis and Control of Systems (MACS). His research interests include fault tolerant control, fault diagnosis and nonlinear systems.

Adel Tellili was born in Tunisia in 1967, he graduated from the Technical University of Karlsruhe, Germany, in 1994. He received the Master degree in Automation in 2003 from the National School of Engineers of Sfax (ENIS), Tunisia and the $\mathrm{PhD}$ degree from the same school in January 2007. He is currently an Assistant Professor at the Higher Institute of Technological Studies at Djerba. He is member at research Laboratory on Modeling, Analysis and Control of Systems (MACS). His research interests include singularly perturbed systems, fault diagnosis and fault tolerant control.

Mohamed Naceur Abdelkrim was born in Tunisia in 1958, he graduated from High School of Technical Education of Tunis, Tunisia, in 1980. He received the Master degree from the same school in 1981 and the PhD degree and "thése détat" from the National School of Engineers of Tunis, Tunisia respectively, in 1984 and 2003. He is currently a professor of automatic control at the National School of Engineers of Gabes. $\mathrm{He}$ is also the head of the research Laboratory on Modeling, Analysis and Control of Systems (MACS). His research interests include robust control, fault tolerant control and singularly perturbed systems. 